\title{
Molecular Binding Energies from Partition Density Functional Theory
}

\author{
Jonathan Nafziger ${ }^{*}$ \\ Department of Physics, Purdue University, 525 Northwestern Avenue, West Lafayette, IN 47907, USA \\ Qin $\mathrm{Wu}{ }^{\dagger}$ \\ Center for Functional Nanomaterials, Brookhaven National Laboratory, Upton, NY 11973
}

\begin{abstract}
Adam Wasserman $\$$
Department of Chemistry, Purdue University, 560 Oval Drive, West Lafayette, IN 47907, USA and Department of Physics, Purdue University, 525 Northwestern Avenue, West Lafayette, IN 47907, USA
\end{abstract}

(Dated: October 31, 2018)

\begin{abstract}
Approximate molecular calculations via standard Kohn-Sham Density Functional Theory are exactly reproduced by performing self-consistent calculations on isolated fragments via Partition Density Functional Theory [Phys. Rev. A 82, 024501 (2010)]. We illustrate this with the binding curves of small diatomic molecules. We find that partition energies are in all cases qualitatively similar and numerically close to actual binding energies. We discuss qualitative features of the associated partition potentials.
\end{abstract}

\section{INTRODUCTION}

Kohn-Sham Density Functional Theory (KS-DFT) [1, 2] provides one of the most useful methods for calculating electronic properties of molecules and materials 3. The accuracy that can be achieved with modern approximations to the xc-functional [4 6], and the efficiency of numerical implemenations [7], make of KS-DFT one of the workhorses of computational quantum chemistry, materials science, and nanotechnology.

A method has recently been proposed that exactly reproduces the results of approximate KS-DFT calculations via self-consistent calculations on isolated fragments [8]. Partition Density Functional Theory (PDFT) is based on the density-Partition Theory (PT) of ref. [9], and is analogous to KS-DFT in that it establishes a map between the physical system of ground-state density $n(\mathbf{r})$, thought of as a collection of interacting fragments, and an auxiliary system of $N_{f}$ non-interacting fragments with ensemble-ground-state-densities $\left\{n_{\alpha}(\mathbf{r})\right\}$ subject to the density constraint $\sum_{\alpha}^{N_{f}} n_{\alpha}(\mathbf{r})=n(\mathbf{r})$. The appeal of this formalism is two-fold: on the one hand, by optimally dividing a complex system into fragments it allows one to build a rigorous foundation for chemical reactivity theory 911 . On the other hand, by solving the molecular Kohn-Sham equations in a different way, it focuses attention on quantities that are amenable to new, different approximations, potentially leading to linear-scaling algorithms for large systems. In that spirit, PDFT is similar to embedded-DFT [12 15]. One such quantity is the partition potential, $v_{p}(\mathbf{r})$, a global molecular property (called reactivity potential in ref. 9], and equivalent in practice to the crystal potential of ref.[16] and the

\footnotetext{
*Electronic address: jnafzig@purdue.edu

†Electronic address: qinwu@bnl.gov

¥Electronic address: awasser@purdue.edu
}

embedding potential of ref. 13). It is used as an additional external potential for each fragment so that the density constraint is satisfied. If the density of the whole is known, as in the original formulation of $\mathrm{PT}, v_{p}(\mathbf{r})$ is simply the Langrage multiplier of the density constraint. Without knowing the whole density, an iterative procedure for deriving $v_{p}(\mathbf{r})$ was designed and illustrated with 1-D models [8, 17. In this work we will show how this procedure is realized for real molecules. This step is of course essential to be able to explore the promise of PDFT on both fronts mentioned before.

The partition potential is the functional derivative of the partition energy, $E_{p}\left[\left\{n_{\alpha}\right\}\right]$, with respect to any of the fragment densities $n_{\alpha}(\mathbf{r})$. This energy is defined as the difference between the molecular ground-state energy $E[n]$ and the sum of the fragment energies $\sum_{\alpha}^{N_{f}} E_{\alpha}\left[n_{\alpha}\right]$.

Kohn-Sham equations can be established so that approximations to $E_{p}\left[\left\{n_{\alpha}\right\}\right]$ yield definite predictions for the ground-state energy and density of the assembly. When the exact $E_{p}\left[\left\{n_{\alpha}\right\}\right]$ is employed implicitly by inversion of molecular KS equations [8, then the exact KSDFT results are recovered without ever having to solve the direct problem for the assembly, but only for the fragments.

Analytical studies on one-dimensional models of heteronuclear diatomics [18 provide an early indication that the fragment dipoles obtained by PDFT are more adjusted to chemical intuition and more transferable than those obtained by other density-partitioning schemes, but more studies are of course needed in real systems.

In this work, by employing the Wu-Yang algorithm 19 for iterative inversion, we demonstrate convergence of the PDFT equations in small diatomic molecules, and discuss qualitative features of partition potentials and partition-energy binding curves for $\mathrm{He}_{2}, \mathrm{H}_{2}$, and $\mathrm{LiH}$. We show that the partition energies and potentials are interesting quantities in themselves, as they can be used as conceptual and interpretative tools. 
First, we summarize the PDFT procedure in SecII providing details of our implementation. Convergence of the PDFT equations is demonstrated in Sec III for the binding curves of $\mathrm{He}_{2}, \mathrm{H}_{2}$, and $\mathrm{LiH}$, along with implications, qualitative features of patition potentials, and $E_{p}$-binding curves (in addition to actual binding curves). Concluding remarks are given in Sec IV.

\section{METHOD}

For the simplicity of discussion, we consider a compound with only two parts ( $A$ and $B$ ), but the method is equally applicable to any number of fragments. We also limit ourselves to fragments with fixed integer number of electrons, as in related recent work on embedding-DFT [13 15, only briefly discussing the issue of chemical potential equalization and fractional electron numbers.

In PDFT, the total energy is expressed as

$$
E[n]=E_{A}\left[n_{A}\right]+E_{B}\left[n_{B}\right]+E_{p}\left[n_{A}, n_{B}\right]
$$

where $n(\mathbf{r})=n_{A}(\mathbf{r})+n_{B}(\mathbf{r})$, and a common functional for $E, E_{A}$ and $E_{B}$ is assumed. The above equation can be viewed as a formal and exact definition of $E_{p}$. To minimize $E$ by variations of fragments' densities, which are built from their own sets of orbitals, we have the following Kohn-Sham equations:

$$
\left[-\frac{1}{2} \nabla^{2}+v_{\alpha}(\mathbf{r})+v_{p}(\mathbf{r})+v_{\mathrm{HXC}}\left[n_{\alpha}\right](\mathbf{r})\right] \phi_{i}^{\alpha}(\mathbf{r})=\varepsilon_{i}^{\alpha} \phi_{i}^{\alpha}(\mathbf{r})
$$

Here, $\alpha$ is a fragment index, i.e $A$ or $B$ in this work. The partition potential $v_{p}(\mathbf{r})$ is common to both fragments, thus has no $\alpha$-index.

$v_{p}(\mathbf{r})$ could be derived explicitly if we knew the functional form of $E_{p}\left[\left\{n_{\alpha}\right\}\right]$. Without an expression for $E_{p}$ as an explicit functional of the $\left\{n_{\alpha}\right\}$, it is also possible to derive $v_{p}(\mathbf{r})$ through an iterative procedure, which was first proposed in ref. 8 and we reiterate here.

Suppose that we are at the beginning of the $k$-th iteration. We obtain all fragment densities $n_{\alpha}^{(k)}$ by solving Eq. 2. We then construct a total pro-molecule density as $\tilde{n}^{(k)}(\mathbf{r})=\sum n_{\alpha}^{(k)}(\mathbf{r})$. Because the effect of $v_{p}(\mathbf{r})$ is to make $\tilde{n}(\mathbf{r})$ the same as the true ground-state density of the whole system $n_{s}(\mathbf{r})$, the difference between $\tilde{n}^{(k)}(\mathbf{r})$ and $n_{s}(\mathbf{r})$ should be used a guidance to update $v_{p}^{(k)}(\mathbf{r})$. For that, we do a constrained search to find the energy of $\tilde{n}^{(k)}$, i.e.

$$
E\left[\tilde{n}^{(k)}\right]=\min _{n \rightarrow \tilde{n}^{(k)}} E[n] .
$$

We employ the direct optimization algorithm of Wu and Yang [19], as used in calculating the frozen density energy in a recently-developed density based energy decomposition analysis [31. Thus we rewrite the above equation as

$$
E\left[\tilde{n}^{(k)}\right]=E_{v}\left[\tilde{n}^{(k)}\right]+E_{\mathrm{HXC}}\left[\tilde{n}^{(k)}\right]+\min _{\Psi \rightarrow \tilde{n}^{(k)}}\left\{T_{s}[\Psi]+E_{\mathrm{X}}[\Psi]\right\}
$$

for a general hybrid functional, where $E_{\mathrm{X}}[\Psi]$ represents a fraction of the HF exchange energy calcualted from a Slater determinant $\Psi$ that is constrained to yield $\tilde{n}^{(k)}$. At the end of this minimization, the effective potential for the molecular Kohn-Sham orbitals is

$$
v_{\mathrm{eff}}(\mathbf{r})=v_{\alpha}(\mathbf{r})+v_{\mathrm{HXC}}\left[\tilde{n}^{(k)}\right](\mathbf{r})-v_{\lambda}(\mathbf{r}),
$$

where $v_{\lambda}(\mathbf{r})$ is just the Lagrange multiplier corresponding to the density constraint and is expanded by a linear combination of atom-centered Gaussian functions. Because $v_{\lambda}(\mathbf{r})$ is used to force the density of the whole system to be $\tilde{n}^{(k)}$, its reverse should have the effect of making $\tilde{n}(\mathbf{r})$ more like $n_{s}(\mathbf{r})$. That is: we can set $v_{p}(\mathbf{r})=-v_{\lambda}(\mathbf{r})$ and start the next iteration of fragment calculations. In practice, we update $v_{p}(\mathbf{r})$ as follows:

$$
v_{p}^{(i)}(\mathbf{r})=v_{p}^{(i-1)}(\mathbf{r})-\theta * v_{\lambda}^{(i)}(\mathbf{r}),
$$

where $i$ is the iteration number, and $\theta$ is a damping factor between 0 and 1 used to control convergence. In our calculation, we have used $\theta=1$ or $\theta=0.25$. The convergency criterion we use is $\left|E\left[\tilde{n}_{k}\right]-E\left[n_{s}\right]\right|<\epsilon$, where $\epsilon=10^{-6}$; this guarantees the converged energy is the same at the ground-state energy. The alternative choice of $\left|E\left[\tilde{n}_{k}\right]-E\left[\tilde{n}_{k-1}\right]\right|<\epsilon$ gives essentially the same results.

\section{RESULTS}

We demonstrate our calculations of $v_{p}(\mathbf{r})$ with three simple examples of diatomic systems: $\mathrm{He}_{2}, \mathrm{H}_{2}$, and $\mathrm{LiH}$. In all calculations, Dunning's aug-cc-pvTz basis set is used for molecular orbitals.

The counter-poise (CP) method is used to account for any Basis Set Superposition Error (BSSE). This approach is crucial in PDFT since $v_{p}(\mathbf{r})$ adds features to the fragment's effective potential directly at the location of the other atom, precisely where the ghost basis functions are added 32 .

The partition potential is expanded by atom-centered Gaussian functions, and each center has five s-type functions, with even-tempered exponents of $2^{n}, n=$ $0, \pm 2, \pm 4$. In the following discussion, we will use several energy terms. Suppose $E_{A}^{0}$ and $E_{B}^{0}$ are the energies of the fragments with no influence of the partition potential; $E_{A}^{p}$ and $E_{B}^{p}$ are their energies with the converged partition potential; and $E_{A B}$ is the energy of the compound. Therefore the binding energy is $E_{\text {bind }}=E_{A B}-\left(E_{A}^{0}+E_{B}^{0}\right)$, and the partition energy is $E_{p}=E_{A B}-\left(E_{A}^{p}+E_{B}^{p}\right)$. We also define the preparation energy as $E_{\text {prep }}=\left(E_{A}^{p}+E_{B}^{p}\right)-\left(E_{A}^{0}+E_{B}^{0}\right)$, which is the energy increase associated with the deformation of fragments. Clearly, $E_{\text {bind }}=E_{\text {prep }}+E_{p}$.

\section{A. Helium Dimer}

Rare-gas dimers are known to be weakly bound due to van der Waals interactions, which are not accurately cap- 
tured by most density-functional approximations. However, because our procedure is general and independent of the exchange-correlation functional, it is not critical to have the correct binding curve. Instead, for a clear demonstration, we use Hartree-Fock exchange only, which is known to be purely repulsive between nonpolar closed-shell systems. As shown in Fig. 1] the binding energy for $\mathrm{He}_{2}$ is all positive and increases rapidly when the internuclear distance is shortened. It also shows that the preparation energy is very small, which means the deformation in He atoms is small, as expected in this system, though it starts to grow when the atoms are too close to each other. The repulsive nature of the interaction means that electron densities are pushed away from each other when the two He atoms are in close contact. Thus the internuclear region has a density decrease, as shown in Fig. 2. In PDFT, this density difference is achieved through deformation of each atom, due to the action of the partition potential. In Fig. 3 we plot $v_{p}$ along the internuclear axis at a few representative internuclear distances. Clearly, $v_{p}$ is most positive in the internuclear region, corresponding to the density deficiency. The magnitude of $v_{p}$ decreases as the internuclear distance increases, until to a point that no $v_{p}$ is needed.

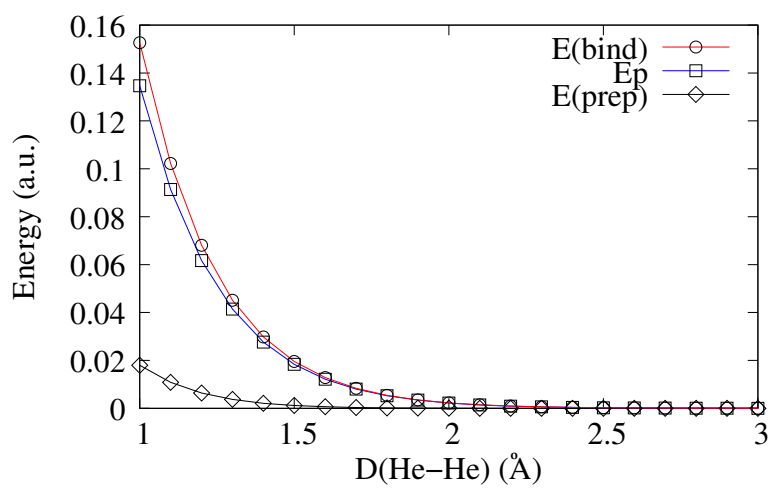

FIG. 1: The Hartree-Fock energies for $\mathrm{He}_{2}$ at different internuclear distances.

It is notable that there are significantly more oscillations in the partition potential than in the density differences. Some of the oscillations are physical. But there are at least two other possible reasons contributing to the oscillations in $v_{p}$. One is pathological with gaussian densities, as nicely explained by Schipper, Gritsenko and Baerends [33. The other is numerical and due to the fact that we expand $v_{p}$ in a finite basis set [34. We have used a small number of functions so as to limit the oscillations caused by the expansion. However, we are unable to use non-gaussian densities yet, which makes it difficult to determine the nature of the oscillations.

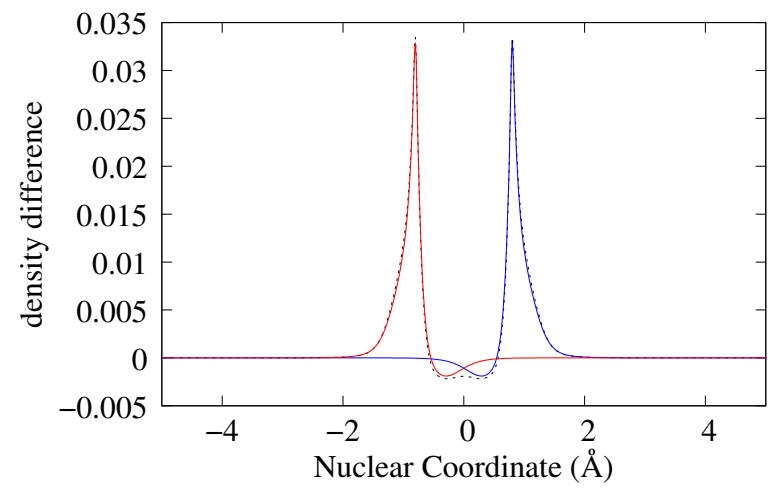

FIG. 2: The density differences in $\mathrm{He}_{2}$ as compared to the original atoms along the line through both nuclei. The total difference (dashed line) is the sum of the deformation in each atom (solid line). The nuclei coordinates are $R= \pm 0.8 \AA$.

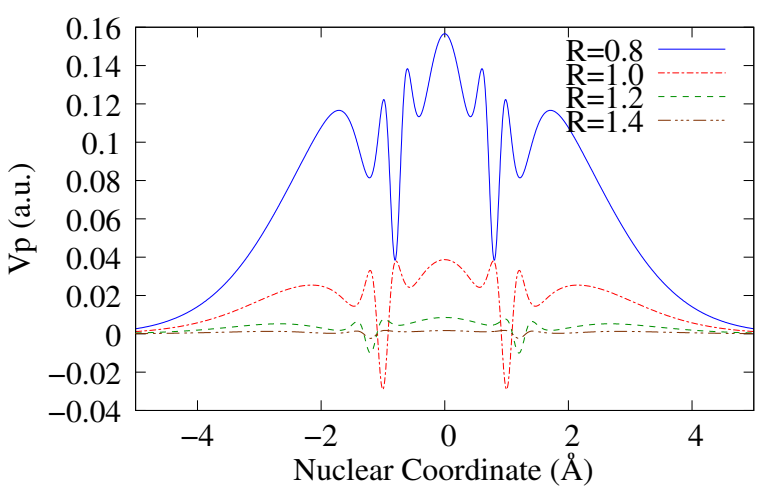

FIG. 3: The partition potentials for $\mathrm{He}_{2}$ at different internuclear distances. The nuclei are at $\pm R$.

\section{B. Hydrogen Molecule}

For the covalently bonded molecule $\mathrm{H}_{2}$, the natural choice of partition is to use two open-shell $\mathrm{H}(\mathrm{OSH})$ atoms. Because their spins are paired up in the molecule, we only consider the total charge density. Mathematically one could also use half-occupied closed-shell $\mathrm{H}$ atoms (CSH) as the fragments, thus without polarizing the spin. We study the energetics of both partitions as a function of the internuclear distance, using the B3LYP approximation to the exchange-correlation functional. For the $\mathrm{H}_{2}$ molecule, we only consider restricted Kohn-Sham (RKS) calculations. It is well-known that a restricted calculation does poorly for large internuclear distances. The erroneous behavior is evident from the binding energy curve when the OSH atoms are used as the reference. As shown in Fig. $4 . E_{\text {bind }}$ approaches a positive value instead of zero. On the other hand, when the CSH atoms are used as the reference, $E_{\text {bind }}$ does go to zero. However, it becomes too large at the optimal bond length. The two binding curves are simply differ- 
ent by a constant shift, and this shift comes from the fact that OSH and CSH have different energies in the B3LYP approximation, while they should be degenerate with the exact functional [35].
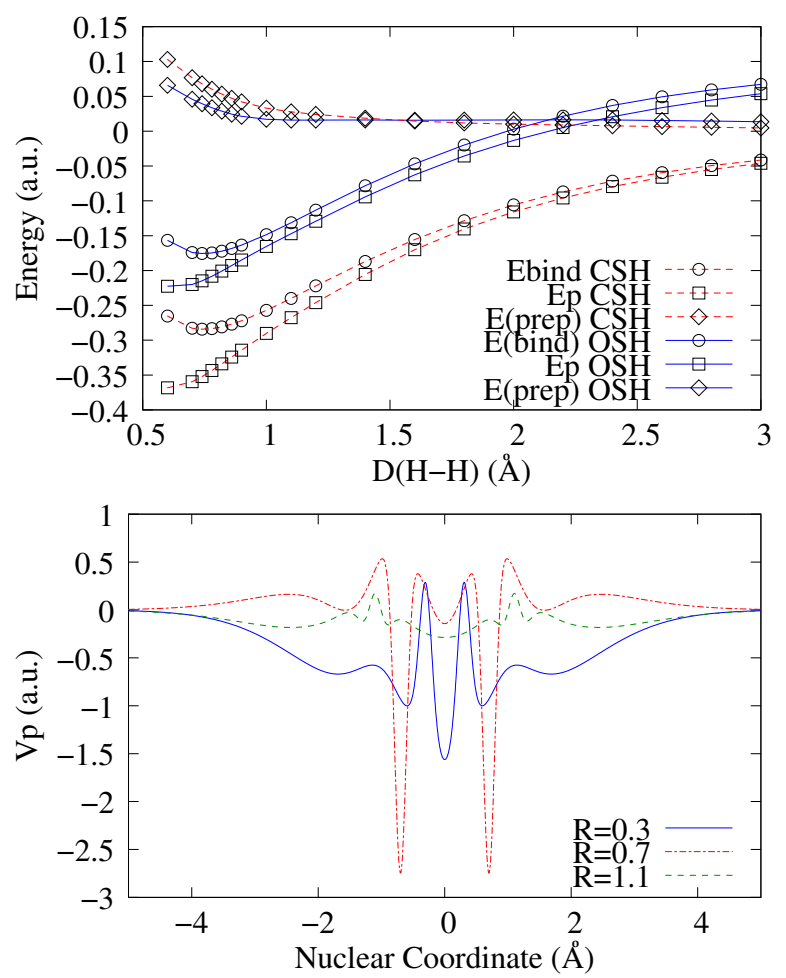

FIG. 4: Top: The B3LYP energies for $\mathrm{H}_{2}$ at different interatomic distances. The optimized bond length is $D=0.743$ A. Bottom: The partition potentials for $\mathrm{H}_{2}$ at different internuclear distances.

In PDFT, the differences in the choice of fragments will not matter if the partition energy can compensate for the difference and yield identical total energy. In our case here, the two $E_{p}$ are indeed quite different. However, the two $E_{p}$ curves differ more than by a simple constant shift. The non-uniform difference can be appreciated by comparing the preparation energies. $E_{\text {prep }}$ of OSH fragments is smaller at short internuclear distances than that of CSH fragments. However, the latter goes to zero at long distances while the former does not. At long distances, a restricted $\mathrm{H}_{2}$ is essentially two half-occupied closed-shell $\mathrm{H}$ atoms, so the asymptotic behavior is not surprising. But it is interesting to see that at short distances, the OSH fragments pay less penalty to make their densities resemble that of the molecule.

\section{Lithium Hydride}

As another example, we consider the heteronuclear LiH. Within the formal partition theory, there is a unique choice of the fragments, with their chemical potentials equilibrated. Achieving equilibration requires treating fragments with fractional number of electrons in the spirit of PPLB [36]. In that case, the number of electrons in a fragment is also a variable to be optimized. Because the partition potential will be different when the fragments change, the optimization of both the partition potential and the number of electrons is mutually dependent and has to be achieved simultaneously. We will treat this complexity in the future. In this work, we simply use fixed fragments and derive the corresponding partition potential.

Without the optimal fragments, we consider all possible partitions. For LiH, there are two possibilities. First, we use neutral atoms. Second, we use $\mathrm{Li}^{+}$and $\mathrm{H}^{-}$. We do the partition at the optimized internuclear distance of $1.59073 \AA$. For the neutral partition, $E_{\text {prep }}=0.053257$ a.u. and $E_{p}=-0.146407$ a.u. For the ionic partition, $E_{\text {prep }}=0.034395$ a.u. and $E_{p}=-0.300161$ a.u. The larger partition energy in the ionic case could be the result of Coulomb attraction. However, the preparation energy is smaller for the ionic partition, suggesting the $\mathrm{LiH}$ bond is closer to an ionic bond than a covalent bond. What is surprising is that the partition potential for the ionic case looks much stronger than the neutral one (Fig. 5), despite causing less distortion in fragment's energies.

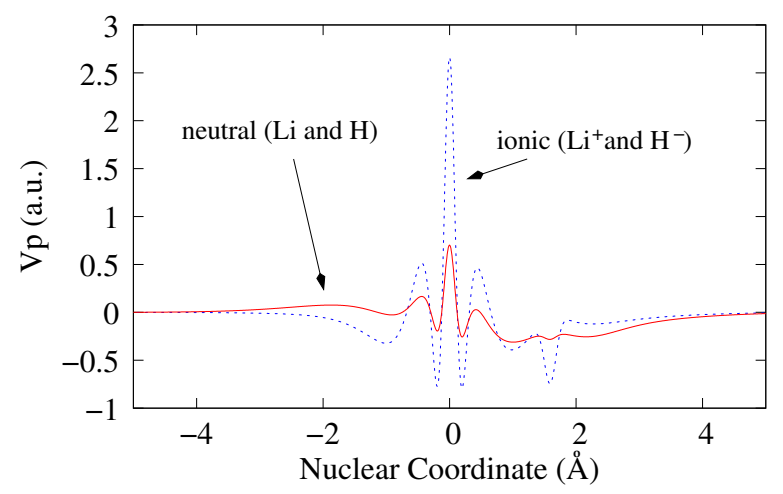

FIG. 5: The B3LYP partition potentials for LiH. The Li atom is at $x=0$ and $\mathrm{H}$ at $x=1.59073 \AA$.

\section{CONCLUDING REMARKS}

Without having to solve directly the $\mathrm{KS}$ equations for the total external potential, we have shown how the PDFT algorithm of ref. 8] provides in practice the same answers via fragment-KS equations. In addition, this method yields fragment densities, fragment energies, and a partition potential that is shared by all fragments such that the sum of their densities reproduces the correct total density.

Although no physical meaning can be attached to a parition potential beyond the one implied by its definition (i.e. that it is the potential common to all fragments 
such that the sum of the fragment densities equals the total molecular density), some generic features of partition potentials seem to go in line with chemical intuition: they are positive when the interaction between fragments is repulsive (case of $\mathrm{He}_{2}$ within Hartree-Fock), and their average magnitude is larger when the interaction between fragments is stronger. Similarly, the strength of the interaction between fragments is loosely measured by the magnitude of the partition energy. No such conclusion can be drawn for the preparation energy, however, as shown for the case of $\mathrm{LiH}$ where a somewhat larger preparation energy is associated with a much smaller partition potential (neutral vs. ionic partition). But the preparation energy can tell us about the character of the bond, an aspect that we plan to study further in future work. The case of $\mathrm{LiH}$ also highlights the need to go beyond integer numbers of electrons in our implementation of PDFT.

PDFT calculations also allow us to look at the dissociation problem from a different angle. For example, we found that open-shell fragments in $\mathrm{H}_{2}$ are preferred at short inter-nuclear separations in the sense that they pay less penalty to make their densities resemble that of the molecule, but close-shell fragments are preferred at long separations. The respective preparation energies cross near the Coulson-Fischer point.

Finally, we point out that from weak $\left(\mathrm{He}_{2}\right)$ to rela- tively strong $\left(\mathrm{H}_{2}\right)$ chemical bonds, partition energies are qualitatively similar to actual binding energies, and numerically close to them (i.e. preparation energies are small in the cases studied). This similarity of $E_{p}$-curves to their corresponding binding curves suggests that approximations of $E_{p}\left[\left\{n_{\alpha}\right\}\right]$ as explicit functionals of the $\left\{n_{\alpha}\right\}$ might be very useful for practical computations. Not only would they provide a direct way to obtain the partition potentials by functional differentiation, circumventing the need of expensive inversion steps; sensible approximations would also lead to energies that are close to actual binding energies. This is analogous to what happens in KS-DFT, whose success is largely due to the fact that the sum of KS orbital energies is typically close to actual ground-state energies in chemical applications.

\section{ACKNOWLEDGMENTS}

We acknowledge support from the Office of Basic Energy Sciences, U.S. Department of Energy, under grant No.DE-FG02-10ER16196. This research was carried out in part at the Center for Functional Nanomaterials, Brookhaven National Laboratory, which is supported by the U.S. Department of Energy, Office of Basic Energy Sciences, under Contract No. DE-AC02-98CH10886.
[1] P. Hohenberg and W. Kohn, Phys. Rev. 136, B 864 (1964).

[2] W. Kohn and L.J. Sham, Phys. Rev. 140, A 1133 (1965).

[3] R.M. Dreizler and E.K.U. Gross, Density Functional Theory (Springer-Verlag, Berlin, 1990); R.G. Parr and W. Yang, Density-Functional Theory of Atoms and Molecules (Oxford, New York, 1989); R.M. Martin, Electronic Structure (Cambridge University Press, Cambridge, 2004).

[4] J.P. Perdew, K. Burke, and M. Ernzerhof, Phys. Rev. Lett. 77, 3865 (1996); 78, 1396 (1997) (E).

[5] J. Tao, J.P. Perdew, V.N. Staroverov and G.E. Scuseria, Phys. Rev. Lett. 91, 14640 (2003).

[6] Y. Zhao and D.G. Truhlar, Theor. Chem. Acc. 120, 215 (2008).

[7] M. Valiev, E.J. Bylaska, N. Govind, K. Kowalski, T.P. Straatsma, H.J.J. van Dam, D. Wang, J. Nieplocha, E. Apra, T.L. Windus, W.A. de Jong, Comput. Phys. Commun. 181, 1477 (2010)

[8] P. Elliott, K. Burke, M.H. Cohen, and A. Wasserman, Phys. Rev. A 82, 024501, (2010).

[9] M.H. Cohen and A. Wasserman, J. Phys. Chem. A 111, 2229 (2007).

[10] M.H. Cohen and A. Wasserman, Isr. J. Chemistry 43, 219 (2003).

[11] M.H. Cohen and A. Wasserman, J. Stat. Phys. 125, 1125 (2006).

[12] C. Huang and E.A. Carter, J. Chem. Phys. 125, 084102 (2006).

[13] C. Huang, M. Pavone, and E.A. Carter, J. Chem. Phys.
134, 154110 (2011).

[14] J.D. Goodpaster, N. Ananth, F.R. Manby, and T.F. Miller III, J. Chem. Phys. 133, 084103 (2010).

[15] J.D. Goodpaster, T.A. Barnes, and T.F. Miller III, arXiv:1102.4028v1 (2011).

[16] P. Cortona, Phys. Rev. B 44, 8454 (1991).

[17] P. Elliott, M.H. Cohen, A. Wasserman, and K. Burke, J. Comp. Theor. Chem. 5, 827 (2009).

[18] Y. Zhang and A. Wasserman, J. Chem. Theory Comput. 6, 3312 (2010).

[19] Q. Wu and W. Yang, J. Chem. Phys. 118, 2498 (2003).

[20] T.A. Wesolowski and A. Warshel, J. Phys. Chem. 97, 8050 (1993).

[21] S.R. Gadre, R.N. Shirsat, and A.C. Limaye, J. Phys. Chem. 98, 9165 (1994).

[22] K. Kitaura, E. Ikeo, T. Asada, T. Nakano, and M. Uebayasi, Chem. Phys. Lett. 313, 701 (1999).

[23] T.E. Exner and P.G. Mezey, J. Phys. Chem. A 106, 11791 (2002).

[24] D.W. Zhang, Y. Xiang, and J.Z.H. Zhang, J. Phys. Chem. B 107, 12039 (2003).

[25] S.H. Li, W. Li, and T. Fang, J. Am. Chem. Soc. 127, 7215 (2005).

[26] L.L. Huang, L. Massa, and J. Karle, Int. J. Quantum Chem. 103, 808 (2005).

[27] V. Deev and M.A. Collins, J. Chem. Phys. 122, 154102 (2005).

[28] R.P.A. Bettens and A.M. Lee, J. Phys. Chem. A 110, 8777 (2006).

[29] J. Rezáč and D.R. Salahub, J. Chem. Theory Comput. 
6, $91(2010)$.

[30] S. Goedeker, Rev. Mod. Phys. 71, 1085 (1999).

[31] Q. Wu, P.W. Ayers, and Y. Zhang, J. Chem. Phys. 131, 164112 (2009).

[32] F. Jensen, Introduction to Computational Chemistry, Wiley (1998).

[33] P.R.T. Schipper, O.V. Gritsenko, and E.J. Baerends, Theor. Chem. Acc. 98, 16 (1997).
[34] T. Heaton-Burgess, F. A. Bulat, and W. Yang, Phys. Rev. Lett. 98, 256401 (2007).

[35] A.J. Cohen, P.Mori-Sánchez, and W. Yang, J. Chem. Phys. 129, 121104 (2008).

[36] J.P. Perdew, R.G. Parr, M. Levy, and J.L. Balduz, Jr., Phys. Rev. Lett. 49, 1691 (1982). 